\title{
Design and Realization of Edible Beans Resource Management Information System
}

\author{
Xiaojie Kang ${ }^{1}$, Yeping Zhu ${ }^{1}$, Huijie Zhang ${ }^{1}$ Hailong Liu ${ }^{1}$ and Jin Gong ${ }^{1}$ \\ ${ }^{1}$ Agricultural Information Institute of Chinese Academy of Agricultural Sciences/Key Laboratory of Agri-Information \\ Service Technology of Ministry of Agriculture, Beijing, China \\ kxiaojie@163.com, zhuyeping @ caas.cn, Corresponding author: zhanghuijie @ caas.cn
}

\begin{abstract}
Supported by the national edible beans Modern industrial technology system in China, Edible Beans Resource Management Information System (EBRMIS) was designed and realized based on Geographic Information System (GIS), which can manage the data standardly, query the data by condition, output the data in chart, as well as query spatial data. The system established by using .NET as the development platform, c\# as development language, and combining with the Teechart technology and the secondary development technology of SuperMap Objects. In this study, EBRMIS was used to analysis edible beans resources in Dali Bai Autonomous Prefecture in Yunan province. The result showed that broad bean was one of the major edible beans resources and the sown area was basically unchanged for between 2011 and 2012; Garden Pea was planted only in Midu County and sown area is less. The result also shown that, from the edible beans yield data, broad bean and Garden Pea had declined, especially for Garden Pea.
\end{abstract}

Key words: Edible Bean; Information system; C\#; GIS

\section{Introduction}

Edible beans belong to legume crop, and can be eaten as food including their grain of dry, fresh and soft pods [1]. In China, edible beans were planted with a long history and there are rich resources. In the recent years, with the improvement of people's living standards, edible beans are changed to important foods to optimize diet structure and to improve the structure of food and nutrition; therefore, consumer demand of them is steadily growing with a strong impetus to the edible beans industrial scale. To solve the current problems of the industry development of China's edible beans, the Ministry of Agriculture combing with the Ministry of Finance set up a special support fund to establish industrial technology system of edible beans to further promote the industry more bigger and stronger[2-4]. Relying on this system, the fixed observation points were set up in the main producing areas of various edible beans to track and to survey the data of cultivation, processing, trade and others of different species of bean, aiming to investigate the basic data of our edible beans resources by comprehensive survey. Based on those data, Edible Beans Resources Management Information System (EBRMIS) was built to management and analysis edible bean production systematically, thus it enables to query and generate relative information of edible beans resources more accurately and rapidly. Due to closely connection between edible bean production, processing, marketing, trading with geographic space, the data has mostly spacial connotation, therefore, adding the spatial information in the course of data analysis can enable data analysis more thorough $[5,6]$.
Geographic Information System (GIS) is a computer system assembling acquisition, storage, management, retrieval, analysis and expression of space data. EBRMIS was developed by merging GIS functionality, as well as combining GIS with management information system functions together, and penetrating geospatial information into the data analysis; thereby it can excavate more intuitive and useful information deeply, and provide precise decision support for edible beans industrial technology system in china. By using EBRMIS, it has important significance in propelling the development of edible bean industrial technology system, adjusting agricultural industrial structure, and promoting agricultural industrial upgrading [7]. At the same time, it also provides the basis for establishing disaster warning of the edible beans and product quality and safety traceability system in the future $[8$, 9].

\section{System Structure}

\subsection{System Overall Structure}

The system overall structure is divided into three layers: human-computer interaction layer, business logic layer and the underlying database layer. Human-computer interaction layer was developed to realize input, query, analyze and output the data by interacting of user and system. Business logic layer was developed by embedding C\# .NET component, SuperMap Objects NET 6R components and statistical analysis components to support logic processing of data. The underlying database layer included the 1:4,000,000 electronic maps of spatial database, attribute database and edible beans resource database (Fig. 1).

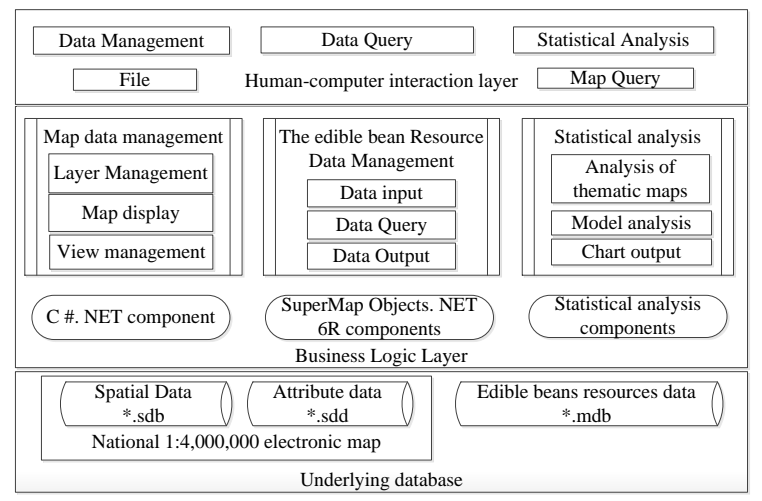

Fig. 1 The structure graph of the Edible Beans Resource Management Information System (EBRMIS) 


\subsection{Database Design}

According to the demand of the system, the database is divided into edible beans resource database and spatial information database. The edible beans resource database is mainly store data from tracking survey at the observation point. The spatial information database was based on 1:4,000,000 electronic map provided by SuperMap Software Co. Ltd. The system was based on Microsoft Access and database access by Structural Inquiry Language (SQL). The edible beans resource database connects with geographic information database by the area code field, which provides a convenient condition to modify and expand the database.

\section{System Function}

\subsection{Data Management}

The users in EBRMIS were grouped by the user authorization: only the administrator was authorized for the data management function, and the user can maintain the original data, input new data and modify change data in data management function. To ensure the analysis of data and the making of computer-aided decision accurately, it is the key point to maintain the database real-time and reliability [10]. In the study, since each observation point has just been established, the data of edible bean resource will be increased gradually, including the number and type etc. The EBRMIS can be input data by manually enter and Excel import.

\subsection{Data Inquiry}

According to the edible beans resource database currently, the data inquiry was divided into bean type inquiry and region inquiry. The bean type inquiry will show the data including cultivation, processing, marketing and other aspects of one bean type in China. The region inquiry will show the all edible beans resources of this region and its associated information [11]. In the EBRMIS, output has two means, one is output in chart based on TeeChart component technology and another is dimensional table based on Excel [12].

\subsection{Statistical Analysis}

The statistical analysis means a process that using statistical methods, following the order from the qualitative quantitative - qualitative, to settle, sort and analyze the collected data and materials, eventually form the analysis report, so as to achieve a more profound understanding of the object of study. Through quantifying the data of edible bean production, marketing, processing and trading on the basis of distinguishing the quantitative boundary to confirm the qualitative change, it can reveal the development state and law of relative edible beans resources data. The achievable indexes of the system are mean, standard deviation, correlation coefficient, the convergence tendency, the discrete degree, and correlation intensity of the describable data. Meanwhile, the system accomplishes the output of statistical data and diagram by utilizing the TeeChart component technology.

\subsection{Map Search}

The map query function of EBRMIS was developed by using the GIS secondary development platform, and SuperMap
Objects.NET 6R, and the latter was developed by SuperMap Software Co. Ltd. with Universal GIS kernel-based (UGC) technology. Universal GIS is characterized by the GIS core technology to realize inside the UGC and to establish interface standards and technical framework access to data outside the UGC, so as to support a variety of data formats to access spatial database and to realize the storage technology, space indexing technology and data query technology(SuperMap SDX+)[13]. With the function of GIS, the system has two means of inquiry: one is from map to attribute data and another is from attribute data to map.

From the map to the attribute data query, the user can click on any area of map within the effective view, and then the system establishes the relationship between spatial database and edible beans resource database via SDX+, finally attribute information of edible beans resource was shown in the map[14].Query from the property to map is the thematic map query. Thematic map focuses on displaying certain kinds of natural phenomena or socio-economic phenomenon in a cartographic region to adapt to special needs. Through analyzing the data by thematic map, combining the attribute data with geographic data, users can have a deeper understanding of the spatial distribution of the data while mining the relations between the attribute data, thus excavate more intuitive and useful information to complete the auxiliary decision-making[17]. Depending on the nature of the attribute data, the system achieves the function of single value thematic maps, segmented thematic maps and statistical thematic map as well as variety of graphics rendering style (size, color, line style, fill, et al.). Single value thematic maps and segmented thematic maps manufacture and analyze thematic maps for single data indicators, and the difference is that the single values thematic maps classify the same data indicators and set a rendering style, focusing on phenomenal qualitative difference, while segmentation thematic maps divide data indicators into multiple range segment in a certain segmented way, and set different rendering style for different range segment, emphasizing the phenomenal quantitative difference. Simultaneously the filter function of segmented thematic maps allows the user to easily query the spatial distribution of different order of magnitude on the map, which contributes to achieving the analysis of the edible bean production, marketing, processing and trade area development. The characteristics of the statistical thematic maps is that it can fabricate thematic maps in view of one data or multiple data indicators, which consequently can achieve statistical analysis of one data indicator and multiple data indicators.

In this research, to show EBRMIS's function, it was used to analyze the edible bean resource data from the county of Eryuan, Dali, Xiangyun and Midu in Dali Bai Autonomous Prefecture in Yunnan province from 2011 to 2012.

Fig. 2 is the thematic map of 2011-2012 edible bean resource area. Overall, broad bean is the local main planting edible bean; Garden Pea has been cultivated in Midu County. From the vertical point of view, broad bean acreage only in Eryuan County have major changes in last two years which 
decreases $100 \mathrm{mu}$, but the other three counties remained unchanged basically. From the lateral point of view, Dali City ranks first in the four counties about broad bean area in 2011, following by Eryuan County, Xiangyun Midu County; Dali City broad bean acreage continued to hold the first in 2012, following by Xiangyun-er source County, Midu County, broad beans in the four counties of the sown area inter relatively little change. The Garden Pea area has no major changes in last two years.

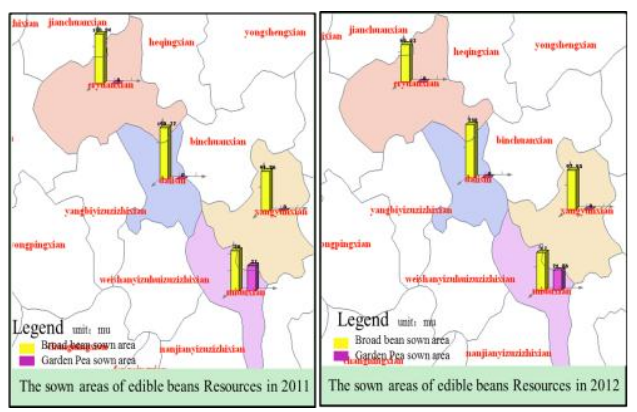

Fig. 2 The thematic map of the edible beans resources sown areas

Fig. 3 shows edible bean yield per mu in 2011, 2012. From the vertical perspective, the other three counties' except Midu County horsebean yield per mu are roughly the same in 2011 and 2012, Midu County's horsebean yield per mu is significantly higher than the other three counties. From the horizontal perspective, Dali City's horsebean yield per mu essentially unchanged, increased slightly. And the other three counties are in decline, Midu County dropped most, declined by about $160 \mathrm{~kg} / \mathrm{mu}$. Peas yield decreased to $460 \mathrm{~kg} / \mathrm{mu}$ in 2012. It shows that natural disasters or other external conditions led to an overall decline in yield per mu of edible beans in 2012 .

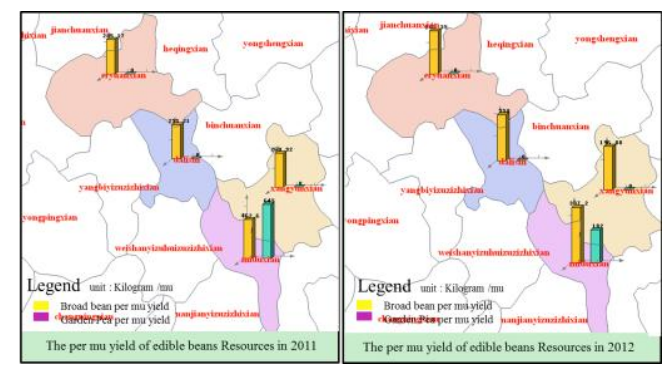

Fig. 3 The thematic map of the per mu yield of edible beans Resources

\section{Conclusion}

Supported by the early establishment of the national edible beans modern agricultural technology system, the EBRMIS was designed and realized based on combination of management information system and geographic information technology to support standardized management, information inquiry, statistical analysis and thematic analysis of production data, marketing data, and Processing data and trading data of edible bean. By using EBRMIS to manage, query and analyze the edible bean resource data of Eryuan,
Dali, Xiangyun and Midu in Dali Bai Autonomous Prefecture in Yunnan, it showed that broad bean in large acreage in four counties, but the per mu yield was far below the average of developing countries and have declined in 2012, which gives suggestions to industrial technology system to pay attention to improve the bean yield level in the next few years. However, there are a lot of factors which have affects on the edible bean production, such as climate, pest and natural disasters, as well as the abnormal changes in market supply and demand. Therefore, the establishment of the information network of edible beans will make information service throughout the whole process of edible bean production, marketing, processing and trade, involving in all aspects of the disaster warning, product circulation, product quality and safety. As a result, it is a major task for the national edible beans industrial technology system in future.

\section{Acknowledgment}

I would like to thank the Dr. zhanguan Hai, Yi Lang and Jie Zhang for their help in the process of the system establishment,and Eric,Huili Yue and Zhen Shi for their valuable help in paper modification.

\section{References}

[1] H, Zhang, Y. Guo, et al, "The analysis of the causes of mung bean price fluctuations in recent years", Issues in Agricultural Economy, no. 4, pp. 30-34, 2012.

[2] X. Chen, "Edible bean production situation and development prospects in Jiangsu Province", Jiangsu Agricultural Sciences, no 5, pp. 4-8, 2009

[3] L. Wang, X. Chen, S. Wang" Advances in Research on Genetic Resources, Breeding and Genetics of Mungbean ", Scientia Agricultura Sinica, val.45, no. 5, pp.1519-1527, 2009

[4]C. Lin, Y Li, S. Wang "Economic value and exploit ways of edible legume crops", Shaanxi Journal of Agricultural Sciences, no 5, pp.29-30, 1999

[5] F. Yang, Y. Zhu, Y. E "Design and Realization of GIS- based Agricultural Economy Information Service System", Chinese Agricultural Science Bulletin, vol. 24, no. 5, pp.429-433, May 2008

[6] W. Lin, "Study on the visualization management of staple agricultural products data information based on GIS technology", Guangdong Agricultural Science, no. 16, pp.185-188, 2012

[7] Y. E, Y. Zhu, "Research and Implementation of an Agricultural Economy Spatial Information Service System", COMPUTER ENGINEERING \& SCIENCE, vol. 30, no. 5, pp.68-71, 2008

[8] Qu, D. Zhuang, "Studies on GIS Based Tracing and Traceability of Safe Crop Product in China", Agricultural Sciences in China, no. 6, pp. 724731, 2007

[9] Yang, J Xie, "Application of GIS used in the crop pest monitoring and forecasting system", Guangdong Agricultural Science, no.10, pp. 200202, 2012

[10] Y. Fan, K. Zhu, "Design and implementation of data management system for facility-based vegetables industry based on B/S structure in Jiangsu province ", Jiangsu Journal of Agricultural Science, vol.27, no.6, pp. 1387-1393, 2011

[11] X. Lin, "The Design and Development of Basic Farm land Geographic Information System",Journal of Chongqing Normal University( Natural Science Edition), vol.22, no.2, pp. 67-71, Jun, 2011

[12] Z. Sun," Development of management information system for rice production based on COMGIS", Transactions of the CSAE, vol.20, no.3, pp.137-140, May, 2004

[13] Y. Zhu," Crops Analysis and Evaluation Thematic Map System Based on GIS", Computer System \& Applications, vol.21, no.4, pp.1-5, 2012

[14] G. Song, "Research and Development of Components Geographic Information Systems", Journal of Image and Graphics, vol.3, no.4, pp.313-317, Apr, 1998 